\title{
Dynamic Clustering Protocol for Data Forwarding in Wireless Sensor Networks
}

\author{
DeepaliVirmani, AkshayJain ,AnkitKhandelwal , Divik Gupta,NitinGarg \\ Department of Computer Science \& Engineering \\ BhagwanParshuram Institute of Technology \\ Delhi, India. \\ deepalivirmani@gmail.com
}

\begin{abstract}
Energy being the very key concern area with sensor networks, so the main focus lies in developing a mechanism to increase the lifetime of a sensor network by energy balancing. To achieve energy balancing and maximizing network lifetime we use an idea of clustering and dividing the whole network into different clusters. In this paper we propose a dynamic cluster formation method where clusters are refreshed periodically based on residual energy, distance and cost. Refreshing clustering minimizes workload of any single node and in turn enhances the energy conservation. Sleep and wait methodology is applied to the proposed protocol to enhance the network lifetime by turning the nodes on and off according to their duties. The node that has some data to be transmitted is in on state and after forwarding its data to the cluster head it changes its state to off which saves the energy of entire network. Simulations have been done using MAT lab. Simulation results prove the betterment of our proposed method over the existing Leach protocol.
\end{abstract}

Keywords: Cluster, Cluster head ,Leach Protocol, Network lifetime.

\section{Council for Innovative Research}

Peer Review Research Publishing System

Journal: INTERNATIONAL JOURNAL OF COMPUTERS \& TECHNOLOGY

Vol 7, No 2 


\section{I .INTRODUCTION}

Sensor networks are composed of small, battery operated sensors, whose main function is to collect and forward the required data to the base stations. WSN's facilitate monitoring and controlling of physical activities from the surveillance areas with better accuracy [1][10]. WSN'shave applications in a variety of fields such as environmental monitoring, military purposes and gathering sensing information in inhospitable locations [1] [10]. A sensor network is an infrastructure comprised of sensing (measuring), computing, and communication elements that gives an administrator the ability to instrument, observe, and react to events and phenomena in the specified environment. The administrator typically is a civil, governmental, commercial, or an industrial entity.

The WSN's are built of "nodes" that range from a few to several hundreds or even thousands, where each node is connected to one (or sometimes several) sensors. Every sensor has three basic units- sensing, radio, and battery, the major constraint being limited energy as the sensor nodes are directly dependent on the battery life [1] [11]. Till battery is charged they are live, so our focus lays on make them live for more amount of time. Consequently, a large number of sensor nodes can be networked to gather sensory data and each sensor performs two main responsibilities, namely, Sensing activities and Routing the sensed data to the base station or a controller (Gateway Sensor

Node). The base station is a master node which is generally fixed and assumed to have uninterrupted power supply or accessible for maintenance. The main responsibility of base station is to collect information from various sensor nodes and process it for further dissemination/actions [2]. The sensor nodes are highly resource constrained. Energy is one of the major issues of thesensor nodes. In wireless sensor network most of the energy is consumed during transmissionand it is further increased with the distance, as energy consumption is directly proportional to thesquare of the distance among the nodes [3]. In order to minimize energy consumption and increaselifetime of the network, distance must also be kept underconsideration [4] [5]. Scalability being another issue of concern as a sensor consists of hundreds or thousands of nodes [6]. In sensor networks these issues are addressed in clusterbased architecture particularly in LEACH [7]. But Leach supports only static cluster formation where cluster head runs out of energy more quickly due to extra responsibility of data aggregation.In this paper we focus on key concern areas with sensor networks like minimizing energy conservation, maximizing network lifetime, real time communication.

\section{LITERATURE SURVEY}

The literature [7] LEACH (Low-Energy Adaptive Clustering Hierarchy) proposed a clustering-based routing protocol that minimizes global energy usage by distributing the load to all the nodes at different points in time. A fixed clustering based approach to prolong the lifetime of sensor networks employing data aggregation is described in literature [4]. The literature [8] RDAG is an approach of Data aggregation by reducing the number of transmissions which is an effective approach to save energy by using the concept of LEACH. The literature [5] and [9] says that energy consumption in a WSN varies with the transmission range. Reducing the transmission range will reduce the power consumed in transmitting a packet toward the neighbors.

The major drawback of LEACH [7] is that it does not support dynamic clustering so to overcome this we propose dynamic clustering protocol that refreshes clusters periodically. Cluster formation is based on residual energy, cost. Our proposed method supports sleep and wait technology where in only energy is consumed only on demand. On demand energy consumption helps in proper network utilization as well as enhancing network lifetime by minimizing energy consumption.

To best of our knowledge none of the previous work has included all these factors in a single work.

\section{A. $\quad$ Proposed Dynamic Clustering Protocol (DCP)}

The proposed DCPproposes formation of clusters depending upon the respective energy level of each node. It introduces the concept of assigning different energy levels to different nodes to balance the responsibility among the nodes with in a cluster. The node with the highest energy level looks for nodes within its transmission range forms a cluster and appoints itself as the cluster head of the cluster formed.Once the cluster head is identified for a cluster, transmission of data takes place from all the other nodes to the cluster head. Cluster head behaves as the data aggregating node for that particular time interval. As soon as nodes forward the data to the cluster head they move to the wait state and remain in the sleep mode until they have something more to transfer. The proposed protocol helps in conserving energy by only allowing cluster head to communicate with other cluster heads. All other nodes except cluster head are in sleep wait so their energy is preserved. Indirectly as energy ispreserved the lifetime of node is increased because lifetime of a node is defined as the time period till it is capable of transmitting data. The data when aggregated [8] at cluster head of each cluster is forwarded to the base station and the energy level of the cluster head is decremented. After a fixed interval of time the energy level of each node in a cluster is revaluatedand compared with other nodes and the node having the highest energy is assigned to be the new cluster head of the cluster. This enables clusterformation even when energy andposition of nodes is changing i.e. dynamic clustering. This leads to an effective utilization of energy of each node in the network. Only the nodes with highest energy levels are used for transmission and the energy of all the other nodes is conserved for future use. 


\section{B. Cluster Formation}

The formation of clustersis based upon the respective energy level of nodes. During cluster formation several parameters such as distance to base station, cluster distance, dissipated energyare taken in simultaneously, none of the previous work encounters them in a single work.

Distanceto Base Station (DB) is the summation of all distances from sensor nodes to the Base station (BS). This distance is defined asfollows:

$$
\begin{aligned}
& \mathrm{D}_{\mathrm{B}}=\mathrm{X}_{1 \mathrm{~s}}+\mathrm{X}_{2 \mathrm{~s}}+\mathrm{X}_{3 \mathrm{~s}}+\mathrm{X}_{4 \mathrm{~s}} \ldots \ldots \ldots \ldots+\mathrm{X}_{\mathrm{ms}} \square \square \square \\
& \mathrm{D}_{\mathrm{B}}=\sum_{\mathrm{i}=0}^{\mathrm{m}}\left(\mathrm{X}_{\mathrm{is}}\right)
\end{aligned}
$$

Where Xis is the sum of distance from the node i to the Base Station. For a larger network, tryto keep this distance minimum because most of the energy will be wasted. However, for a smallernetwork the nodes near to base station directly send the information may be an acceptableoption.

Cluster Distance (DC) is the summation of the distances from the member nodes to the cluster head and thedistance from the cluster head to the BS. For a cluster with $\mathrm{k}$ member nodes, thecluster distance DC is defined as follows:

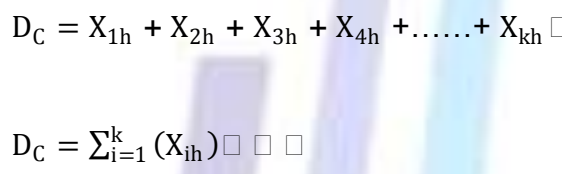

Where Xih is the distance from node $\mathrm{i}$ to the cluster head. For a cluster that has large number of spreads nodes, the distance among the nodes i to cluster head will be more and the energy consumptionwill be higher. So keep the cluster size small to reduce energy dissipation and DC should not betoo large. This metric will keep control the size of the clusters.

Theequation(5) shows the total distancesTdistfrom cluster member i to cluster head andfrom cluster head to BS.

$\mathrm{T}_{\text {dist }}=\sum_{\mathrm{i}=1}^{\mathrm{m}}\left(\mathrm{x}_{\mathrm{ih}}+\cdots \ldots \ldots+\mathrm{x}_{\mathrm{ihb}}\right)$

The total dissipated energy "Etotal" is the total energy dissipated to transfer the aggregatedmessages from the cluster to the BS. For a cluster with $\mathrm{k}$ member nodes, the total dissipatedenergy can be calculated follows:

$\mathrm{E}_{\text {total }}=\sum_{\mathrm{i}=1}^{\mathrm{m}}\left(\mathrm{E}_{\mathrm{Tich}}+\mathrm{K} \times \mathrm{E}_{\mathrm{R}}+\mathrm{E}_{\mathrm{Tchb}}\right)$

The first part of equation (6) shows the energy consumed to transmit messages from member nodesto the cluster head. The second part showsthe energy consumed to transmit aggregatedmessages by cluster head to BS.

The salient features of DCP include:

Dynamic clustering

Highest energy node selected as cluster head

Prolonged Lifetime

Sleep and wait

Energy balancing

Time to time refresh of clusters

\section{IV.ALGORITHM}

initialise():- Parameters of the node are assigned values here.

cal_distance():- Calculates distance of each node from all other nodes and stores them in the distance matrix. 
energy_max():- Determines the node with max energy from the nodes which are not a part any cluster yet and returns the id of that node.

status_update():- Marks the status of the node as active or inactive.

Make_cluster():

1 Loop1: For $\mathrm{i}=1$ to No_of_Nodes

2 Do

3 Emax_id=energy_max()

4 if(emax_id!=-1) then

5 subsink[i]=node[emax_id].id

6 node[emax_id].ss_flag $=1$

7 increment cluster_id

8 node[emax_id].cluster_no=cluster_id

9 loop2: For $\mathrm{j}=1$ to No_of_Nodes

10 Do

11 if(node[j].cluster_no==0) then

12 if(distance[emax_id][j]<=range) then

13

14

15

node[j].cluster_no=cluster_id

end loop2

end loop1

In this algorithm the node with the highest energy level looks for nodes within its transmission range to form a cluster and appoint itself as the cluster head of the cluster formed. The process goes on till all the nodes are not covered under some or the other cluster.

$\mathrm{DCP}()$ :

1 while true,

2 do

$3 \quad$ while(subsink[n]!=0)

4 do

5 if(energy_val[subsink[n]-1]<=0) then

$6 \quad$ print NETWORK DEAD

$7 \quad$ return false

8 decrement energy_val[subsink[n]-1] by 10 units

9 increment $\mathrm{n}$

10 end while

11 loop1: for time=1 to REFRESH_TIME

12 do

13 wait for 1 time unit

14 status_update():

15 loop2: for $\mathrm{i}=1$ to No_of_Nodes 


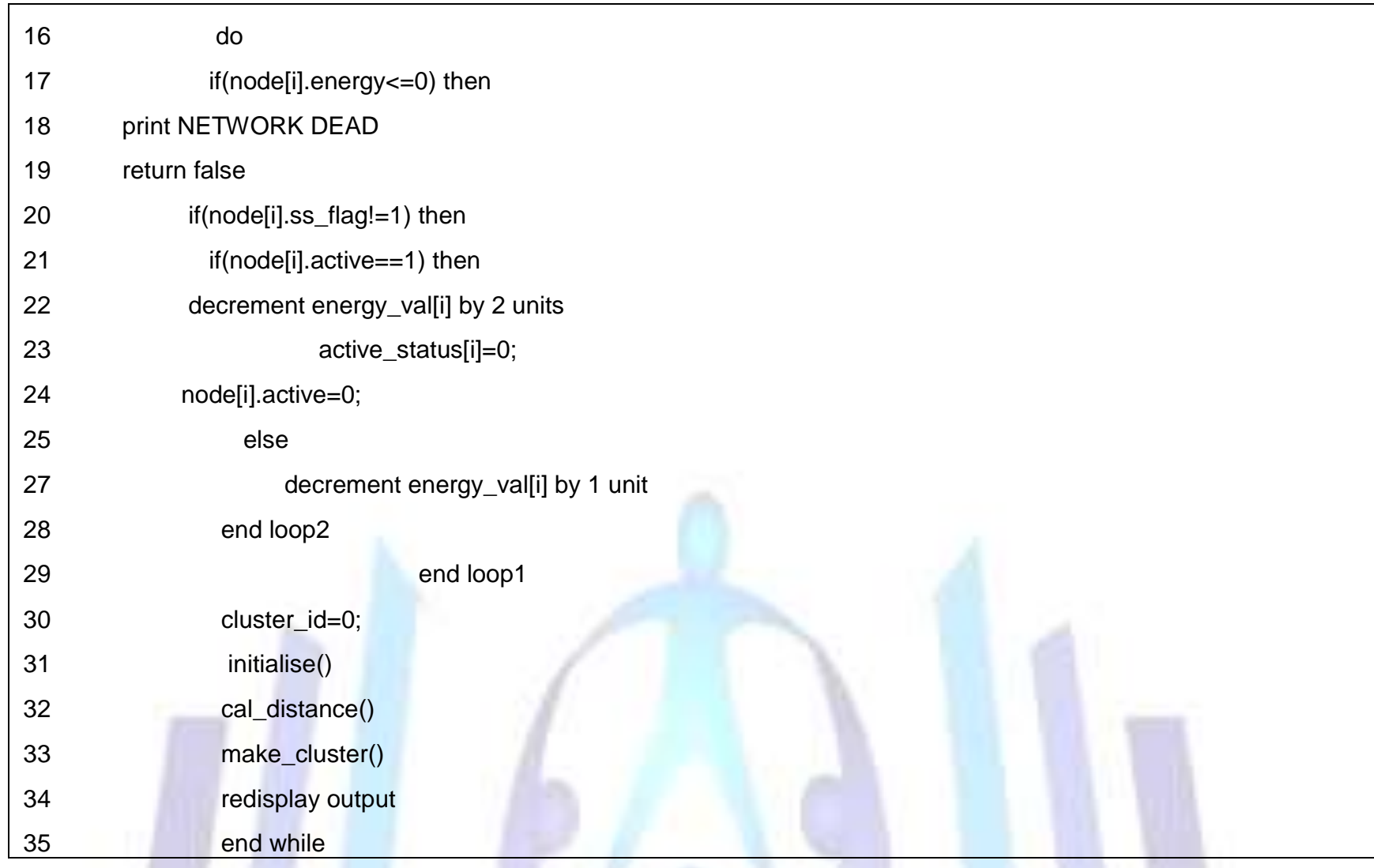

In this algorithm transmission of data takes place from all the other nodes to the cluster head. Cluster head behaves as the data aggregating node for that particular time interval. As soon as nodes forward the data to the cluster head they move to the wait state and remain in the sleep mode until they have something more to transfer. After some interval of time called refresh time clusters are reformed and the process repeats.

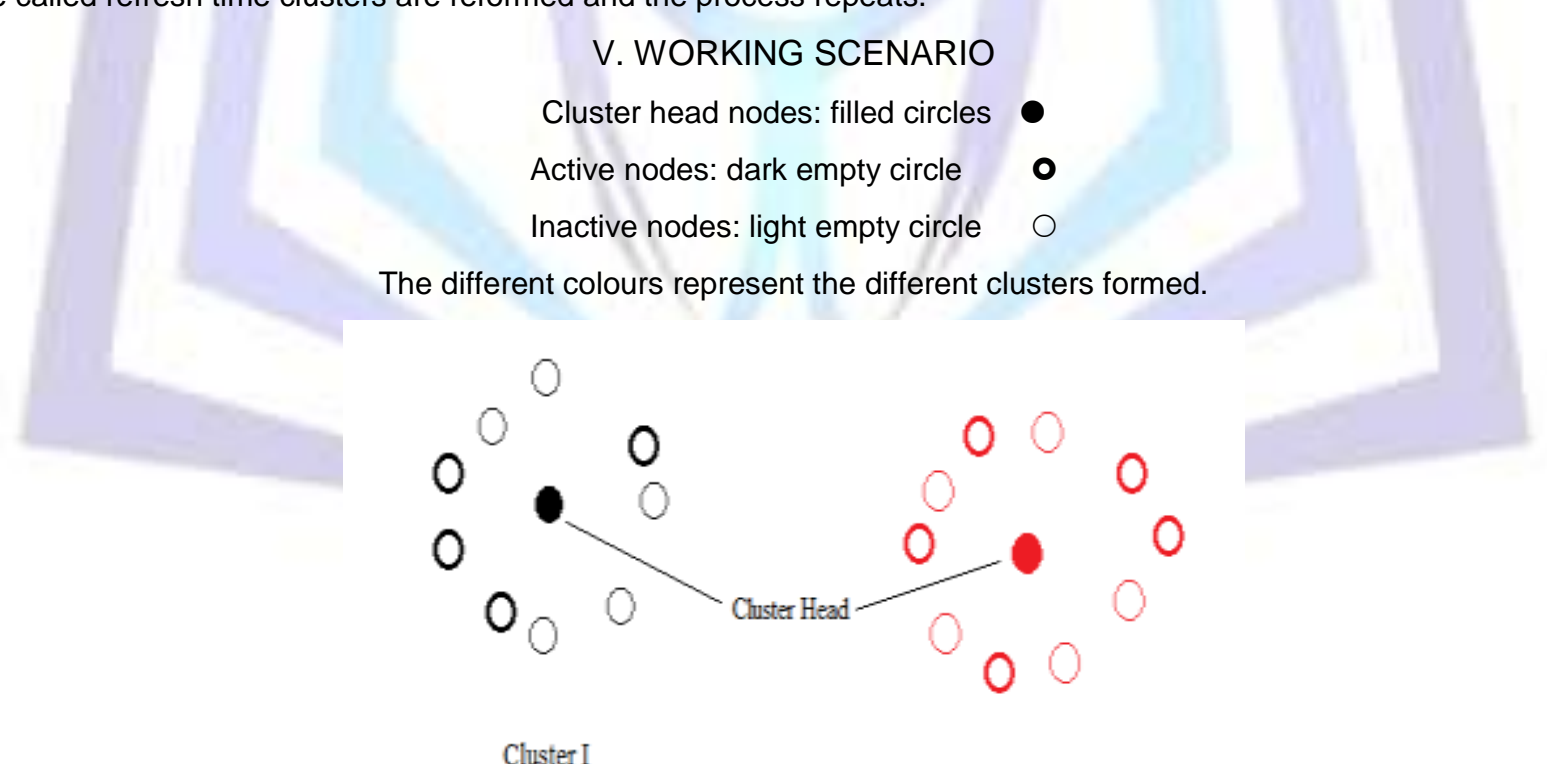

Cluster II

Fig.1.ClusterRepresentation 


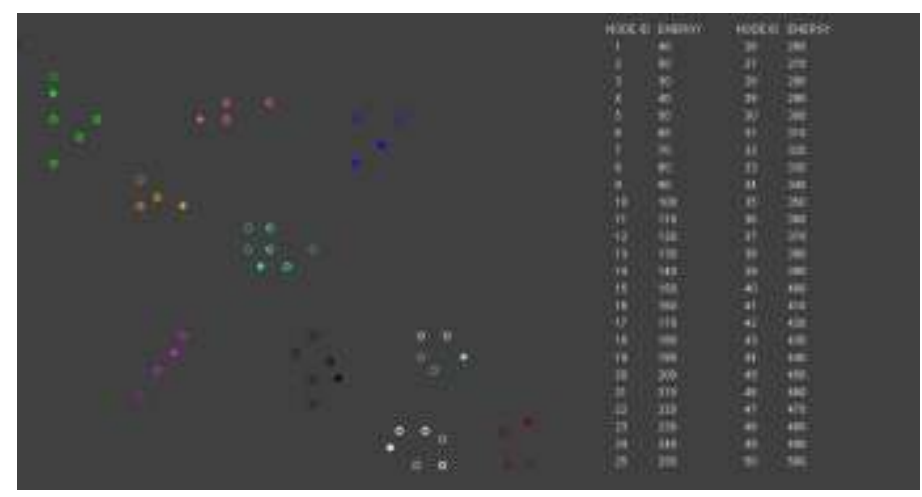

Fig.2.1.Initialclusters(Activestage)

Fig. 2.1 represents that clusters are formed on the basis of energy levels and the node with the highest energy level looks for nodes within its transmission range and forms a cluster and appoints itself as the cluster head of the cluster formed. The filled circle in every cluster represents the cluster head of that cluster and dark empty circle represents the active node and light empty circles are the inactive nodes. The energy values of all the nodes are also displayed along with the nodes ids.

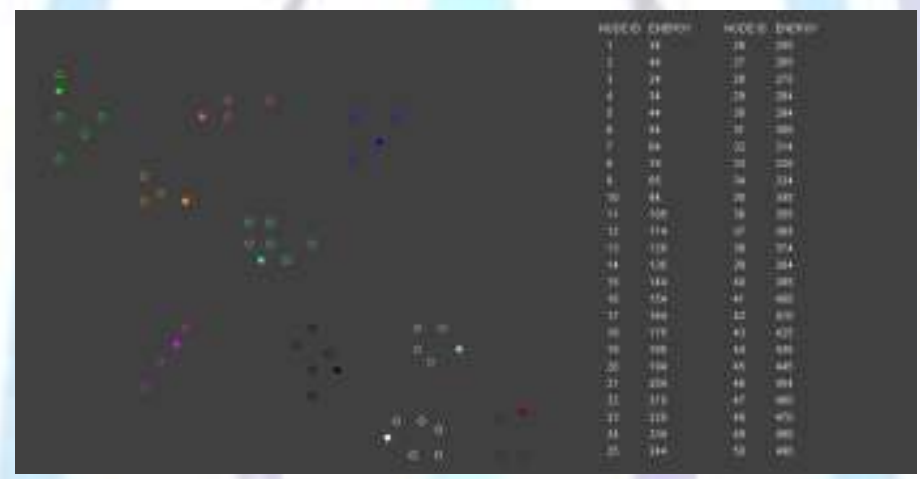

Fig.2.2.Inactivestage

Fig. 2.2 represents the status of the clusters and nodes after nodes have finished transmission of data and all the nodes are in inactive state. The energy values of all the nodes are also displayed along with the nodes ids.

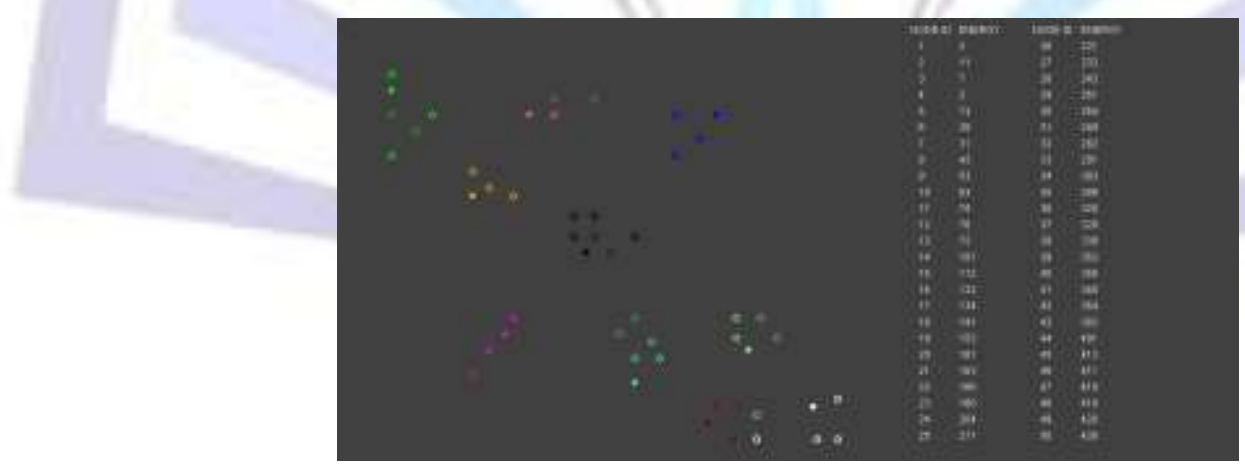

Fig.2.3.Newclustersafterrefreshtime(Active stage)

Fig. 2.3 represents the nodes after refreshing time when new clusters are formed and new cluster heads are appointed. The new decremented energy values of the nodes are displayed. 


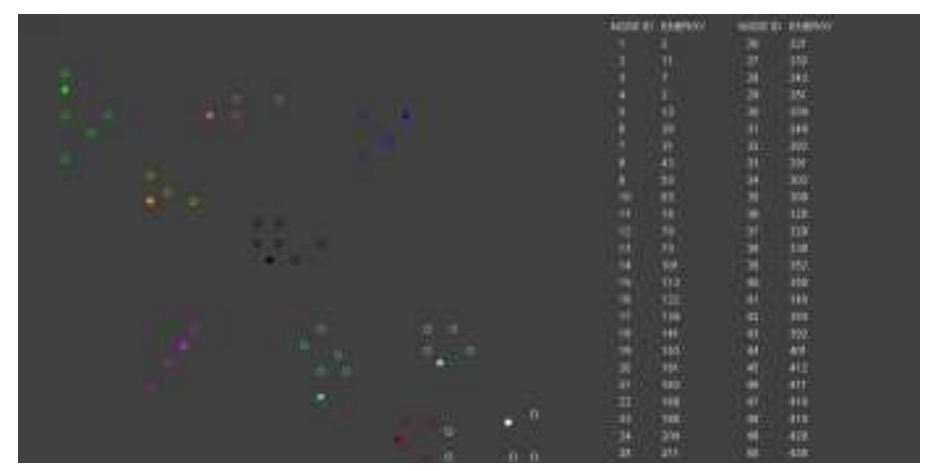

Fig.2.4. Inactivestage

Fig. 2.4 represents the status of the clusters and nodes after nodes have finished transmission of data and all the nodes are in inactive state. The energy values of all the nodes are also displayed along with the nodes ids.

\section{VI.SIMULATION RESULTS}

We simulated and evaluated the performance of the proposed protocol with the existing Leach. All the simulations are done in MATLAB in order to validate the efficiency of proposed protocol.

\begin{tabular}{|l|l|l|}
\hline No. & Parameter & Value \\
\hline 1. & Simulation Area & $1000 \times 1000$ \\
\hline 2. & No. of nodes & 450 \\
\hline 3. & Radio Propagation Model & Two way ground \\
\hline 4. & Channel Type & Wireless Channel \\
\hline 5. & Antenna Model & Antenna/Omniantenna \\
\hline 6. & Energy Model & Battery \\
\hline 7. & Round Duration Time & $10 \mathrm{~s}$ \\
\hline & & 1 \\
\hline 8. & Initial Energy of each node & $0.5 \mathrm{~J}$ \\
\hline
\end{tabular}

Table1.Simulation Parameters

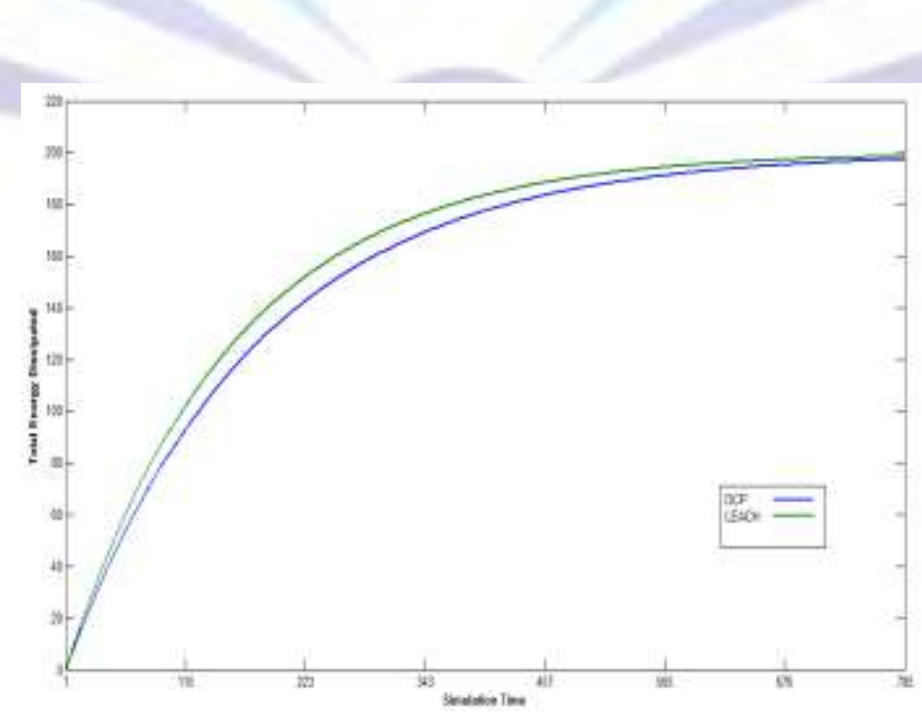

Fig.3.1.TotalenergydissipatedinLEACHandourscheme(DCP) 
Fig. 3.1 shows the total energy consumption of the network over simulation time. The simulations prove DCP to be better than Leach in terms of energy consumption. At the start of simulation performance of Leach and DCP is comparable but as the simulation time proceeds DCP conserves a lot of energy due to its sleep and wait technology. Asonly the active nodes consume energy and nodes after forwarding data to cluster head go to sleep mode.

Fig. 3.2 shows the proposed method compared with the traditional Leach protocol in terms of network lifetime. As the energy consumption of DCP is high as compared to Leach and energy consumption is directly proportional to network lifetime. As node is alive till it runs out of energy so if energy is conserved its lifetime will increase which indirectly increases network lifetime

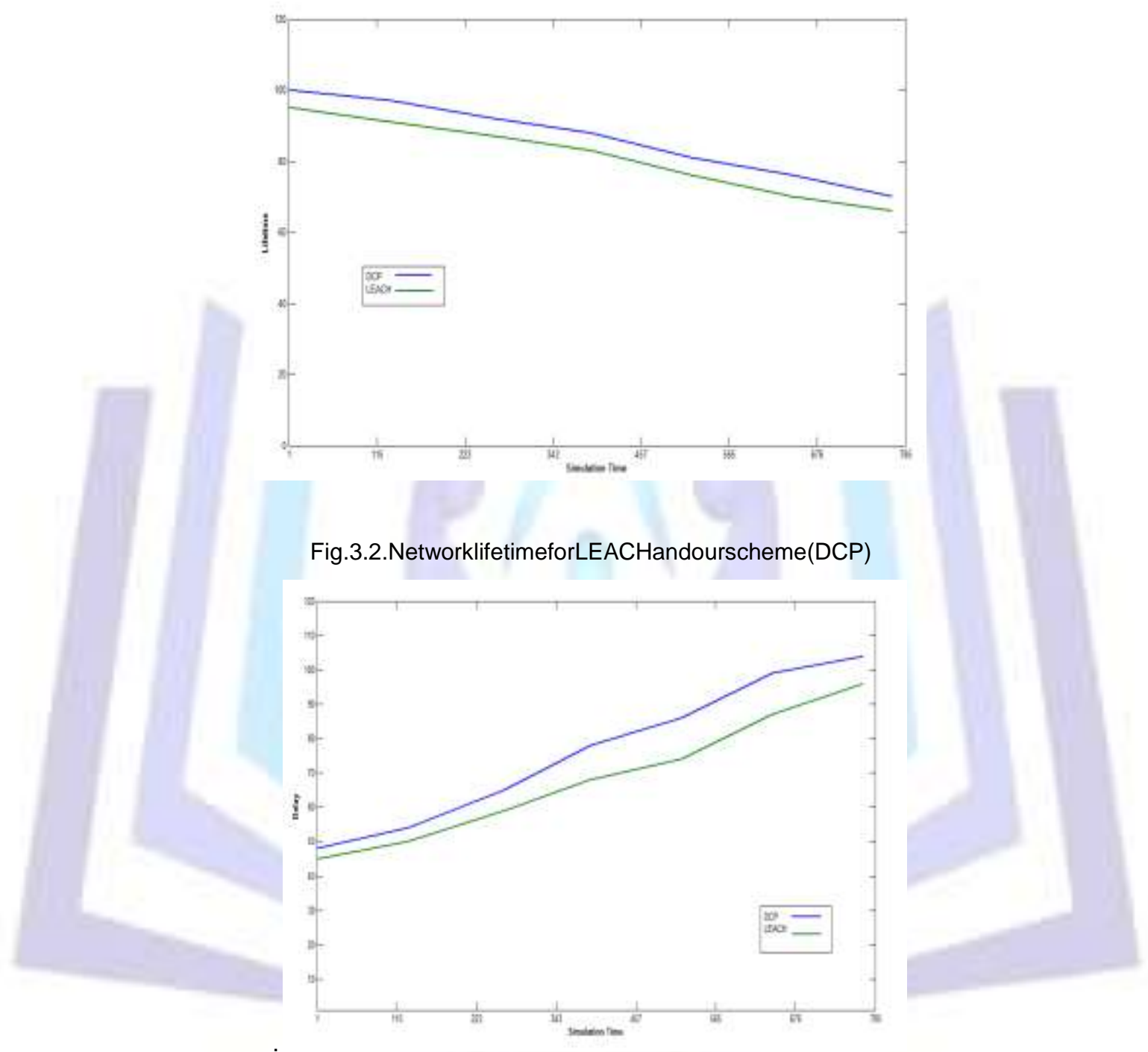

Fig.3.3.Delayin LEACH and our scheme(DCP)

Fig. 3.3 shows the proposed method compared with the traditional Leach protocol has a longer delay than Leach protocol primarily because DCP supports dynamic clusters and clusters are refreshed periodically. Selection of cluster head and refreshment of cluster increases delay but delay is kept proportional to real time delay (supporting real time communication) same is shown by simulation results.

\section{CONCLUSIONS}

Clustering is a useful topology-management approach to reduce the energy consumption and exploit data aggregation in wireless sensor networks. In this paper we have focused and proposed dynamic clustering where clusters are refreshed periodically and cluster head is selected accordingly. The existing protocols LEACH and RDAG support static clustering and cluster head is fixed in the entire scenario. Cluster head being used as the data aggregator node runs out of energy. So in this paper we have proposed a dynamic clustering protocol (DCP) that supports dynamic clustering with support of sleep and wait technology, where the node that needs to transmit the message is only in wake state after forwarding the message the node changes the state to sleep. By this a lot of energy is conserved enhancing network lifetime indirectly. We have simulated and compared the results in MAT lab of our proposed protocol DCP with the existing LEACH. 
Simulation results our proposed protocol are better in terms of energy conservation and enhancement of network life time as sleep and wait technology enhances the lifetime of nodes. Delay is increased in our DCP but still it is in real time communication limits so increased delay is not a harmful factor. In future we will compare the proposed DCP on more existing parameters. As well as we will make DCP work in real time parameters to support real time communication.

\section{VIII.REFRENCES}

Mehdi Saeidmanesh, MojtabaHajimohammadi, and Ali Movaghar, "Energy and Distance Based Clustering: An Energy Efficient Clustering Method for Wireless Sensor Networks", World Academy of Science, Engineering and Technology , USA, Vol 3, 2009, pp 212- 230.

Jamal N. Al-Karaki, RazaUl-Mustafa, Ahmed E. Kamal, "Data aggregation in wireless sensor networks- exact and approximate algorithms", International Workshop on High-Performance Switching and Routing, Phoenix, AZ, April 2008, pp 23-31.

NiveditaRajani, "Energy Efficient, Reliable Routing Protocol for Wireless Sensor", International Journal of Computer Science and Technology, Vol 1, Issue 4, June 2008, pp 6-14.

I.F. Akyildiz, W. Su*, Y. Sankarasubramaniam, E. Cayirci, "Wireless sensor networks: a survey", Elsevier Science Journal of Computer Networks, 2002, pp 393-422.

Wendi RabinerHeinzelman, AnanthaChandrakasan, and HariBalakrishnan, "Energy-Efficient Communication Protocol for Wireless Micro sensor Networks", 33rd Hawaii International Conference on System Sciences, 2009, pp 63-71.

Mohammad HosseinYeganeh, HamedYousefi, NaserAlinaghipour and Ali Movaghar, "RDAG: A Structure-free Real-time Data Aggregation Protocol for Wireless Sensor Networks", IEEE International Conference on Embedded and Real-Time Computing Systems and Applications, July 2010, China, pp 94-102

.J. Zhang, Q. Wu, F. Ren, T. He, and C. Lin., "Effective data aggregation supported by dynamic routing in wireless sensor networks", IEEE International Conference on Communications (ICC'10), May 2010, pp 55-64

.C.Jaikaeo and C.Chien-Chung Shen, "Sensor Information Networking", International Journal of Wireless networls, Wiley Publication, Vol 12, Issue 3, 2005, pp 22- 47

.P.Yen and C.L.Hameed, "Performance Analysis on Energy Aware MAC protocol for Wireless Sensor Network", International Conference on Network and Electronics Engineering IPCSIT vol.11, (2011) IACSIT Press, Singapore, pp 4853.

Ashish Xavier Das, Charlie Eapen, A. Ashok, SudhanshuTripathi, RidhaMabrukShadi, "Dynamic Event Based Energy Efficient Routing Protocol For Wireless Sensor Networks (WSNs)", International Journal of Engineering and Advanced Technology (IJEAT), Volume-1, Issue-6, August 2012, pp 31- 45.

ArchanaBharathidasan, Vijay AnandSaiPonduru, "Sensor Networks: An Overview ", IEEE International Conference on latest trends in Computer Science, Indonesia, Oct 2010, pp 256- 278. 